\title{
Schedulability Analysis and Priority Assignment for Global Job-Level Fixed-Priority Multiprocessor Scheduling
}

\author{
Hyoungbu Back, Hoon Sung Chwa, Insik Shin \\ Dept. of Computer Science, KAIST, Republic of Korea \\ insik.shin@cs.kaist.ac.kr
}

\begin{abstract}
Unlike uniprocessor scheduling, EDF (categorized into job-level fixed-priority (JFP) scheduling) shows relatively poor performance on global multiprocessor scheduling. As no other global JFP multiprocessor algorithms are illuminated beyond EDF, this work proposes one, called EQDF (earliest quasi-deadline first), as a generalization of EDF. We define the quasi-deadline of a job as a weighted sum of its absolute deadline (capturing "urgency") and its worst case execution time (capturing "parallelism") with a system-level control knob to balance urgency and parallelism effectively. This paper then seeks to explore how it can improve the schedulability of global JFP scheduling. In addition to providing a new schedulability analysis for $E Q D F$ scheduling, it addresses the problem of priority assignment under $E Q D F$ by controlling the system-level control knob. It presents optimal and heuristic solutions to the problem subject to our proposed EQDF analysis. Our empirical results show the proposed heuristic solution outperforms EDF significantly, giving close to optimal results.
\end{abstract}

\section{Introduction}

Real-time scheduling has been extensively studied for several decades over various scheduling categories. In general, priority-driven preemptive scheduling algorithms can fall into three categories: task-level fixed-priority (TFP), job-level fixed-priority (JFP) ${ }^{1}$, and job-level dynamicpriority (JDP) algorithms. A TFP algorithm assigns the same priority to all the jobs in each task, and the priority of each task is fixed relative to other tasks. Good examples include RM (rate-monotonic) and DM (deadline-monotonic). A JFP algorithm can assign different priorities to the individual jobs in each task, but the priority of each individual job is fixed relative to other jobs. A good example is EDF (earliest deadline first). Under JDP scheduling, each job is assigned a priority that can change dynamically during the

\footnotetext{
${ }^{1}$ This category is also called task-level dynamic-priority in the literature [25]. However, we use the term JFP in order to emphasize the static nature of the priority of an individual job.
}

job's execution. A good example is LST (least-slack-time first).

Those three categories have been well studied in uniprocessor scheduling. DM [23], EDF [24], and LST [18] are proved to be optimal in each of the three categories, respectively. However, they have not been developed as maturely yet in multiprocessor scheduling as in uniprocessor scheduling. In particular, JFP multiprocessor scheduling has not been yet much explored beyond EDF.

A considerable amount of work has been made to study TFP multiprocessor scheduling. Many heuristic schemes were proposed for priority assignment in this category [3, 16], and an optimal priority assignment subject to some given schedulability test was presented under some conditions [16].

There has been a growing attention to JDP multiprocessor scheduling. The pFair algorithm [12] is known as optimal for implicit-deadline task systems (deadline equal to task period) but no longer optimal for constrained-deadline task systems (deadline no larger than task period). Recent studies showed that JDP algorithms, such as EDZL (EDF until zero-laxity) [22, 9], FPZL (fixed-priority until zerolaxity) [17], and LST [20], outperform TFP and JFP algorithms. In general, JDP algorithms incur relatively significant runtime scheduling overheads, for examples, with frequent context switches and/or with keeping track of laxity dynamically, compared to TFP and JFP algorithms.

A substantial body of research was made to investigate global EDF multiprocessor scheduling. Unlike uniprocessor scheduling, EDF is no longer optimal but exhibits significantly lower performance in multiprocessor scheduling $[19,7]$. Despite this, however, little work has been reported to explore JFP scheduling beyond EDF on multiprocessors. This motivates the research described in this paper with a curiosity to understand how effective JFP scheduling algorithms can be on multiprocessors. We believe that EDF performs poor on multiprocessors because it assigns priority with a sole focus on the deadline constraints (or "urgency") but neglecting the "parallelism" aspect of multiprocessor platforms. According to a few studies [3, 16, 20], assigning a higher priority to the job with a larger execution time helps to maximize the potential for concurrency. This is because it is generally easier to schedule on multi- 
processors a larger number of jobs with shorter remaining execution times than a smaller number of jobs with longer remaining execution times even though they have the same remaining execution times in total. Inspired by this, we define the quasi-deadline $\left(q_{i}\right)$ of an individual job $\left(J_{i}\right)$ as a weighted sum of its absolute deadline $\left(d_{i}\right)$ and its worstcase execution time $\left(C_{i}\right)$ such that $q_{i}=d_{i}-k C_{i}$, where $k$ is a real value that the system designer configures statically and/or the system determines dynamically. We then introduce a new JFP scheduling algorithm, called EQDF (earliest quasi-deadline first), that assigns priority to jobs according to their quasi-deadlines. EQDF is a generalization of EDF; EQDF becomes EDF with $k=0$.

We present a new schedulability analysis for EQDF scheduling, and this facilitates the comparison between the only, well-known JFP algorithm, EDF, and a new JFP algorithm, EQDF, on the basis of schedulability. A critical factor to improve the effectiveness of EQDF scheduling is quasi-deadline assignment. In this paper, we consider the $k$-controlled quasi-deadline assignment that determines the value of $k$ to make a given task set deemed schedulable according to the proposed EQDF schedulability test. A naive approach of examining all possible values of $k$ is prohibitively expensive and even inapplicable to continuous values of $k$. We thereby present an optimal solution algorithm, called OQDA- $k$, that finds a solution value of $k$, if there exists any. Our empirical results show that the proposed EQDF optimal solution does not simply dominate EDF but outperforms it significantly. Our EQDF solutions find $40 \%-60 \%$ more task sets deemed schedulable than the state-of-the-art EDF analysis. Our empirical results also reveal that the OQDA- $k$ algorithm employs a certain amount of running time, leaving the algorithm only suitable for design time. Thereby, we present a heuristic solution to the problem as well. A key factor to performance is where and how densely the heuristic algorithm examines $k$ values. Based on thorough understanding of empirical results, we are able to reduce the search space of the heuristic solution quite effectively. Our simulation results show that the proposed heuristic algorithm can find a solution close to optimal ( $1 \%$ loss of optimality) while reducing running time by two orders of magnitude. It is also shown that the heuristic algorithm is able to find $40 \%-57 \%$ more task sets deemed schedulable than EDF with a comparable running time.

Related Work. Several schedulability tests for global EDF scheduling of sporadic task systems have been developed [7, 19, 10, 14, 26]. Baker [7] developed a general strategy using the notion of processor load for determining the schedulability of sporadic task sets. Baker's test computes a necessary amount of processor load to cause a deadline to be missed and take the contra-positive of this to derive a sufficient schedulability test. Building upon Baker's work, Bertogna et al. [14, 26] developed a sufficient schedulability test for any work conserving algorithms based on bounding the maximum workload in a given interval. Bertogna et al. extended this test via an iterative schedulability test that calculates a slack value for each task, and then uses this value to limit the amount of carry-in workloads.

Priority assignment has been studied in global TFP multiprocessor scheduling. Audsley [4, 5] developed an optimal priority assignment (OPA) policy for some given test on uniprocessor platforms. Davis and Burns [16] showed that Audsley's OPA algorithm is applicable to the multiprocessor case when a given test satisfies some conditions. Several heuristic priority assignments are devised [15, 1, 3, 16] for global TFP multiprocessor scheduling, and some of them are related to our notion of quasi-deadline. Andersson and Jonsson [3] designed the TkC priority assignment policy which assigns priorities based on $\left(T_{i}-k \cdot C_{i}\right)$, where $T_{i}$ is a task period and $k$ is a real value computed on the basis of the number of processors. Davis and Burns [16] developed the D-CMPO policy that assigns priorities according to $D_{i}-C_{i}$, where $D_{i}$ is a task's relative deadline. However, no work addresses optimal priority assignment in global JFP multiprocessor scheduling.

Organization. Section 2 presents system models and terminologies. Section 3 derives a new, interference-based schedulability condition for EQDF scheduling on the basis of understanding of worst-case inter-task interference scenarios under EQDF. Section 4 formulates the $k$-controlled quasi-deadline assignment problem and proposes optimal and heuristic solutions. Section 5 provides empirical results that illuminate the characteristics of optimal solutions thoroughly and evaluates the effectiveness of our EQDF solutions. Section 6 concludes with future work.

\section{System model and terminology}

Task model. In this paper, we assume a sporadic task model, where a task $\tau_{i} \in \tau$ is specified as $\left(T_{i}, C_{i}, D_{i}\right)$ such that $T_{i}$ is the minimum separation, $C_{i}$ is the worst-case execution time requirement, and $D_{i}$ is the relative deadline $\left(C_{i} \leq D_{i} \leq T_{i}\right)$. A task utilization $U_{i}$ is defined as $C_{i} / T_{i}$, and the system utilization $U_{\text {sys }}$ is defined as the total utilization of a task set. A task $\tau_{i}$ invokes a series of jobs, each separated from its predecessor by at least $T_{i}$ time units. When a job $J_{i}^{h}$ of task $\tau_{i}$ has a release time $r_{i}^{h}$, its absolute deadline $d_{i}^{h}$ is given as $d_{i}^{h}=r_{i}^{h}+D_{i}$. The scheduling window of a job $J_{i}^{h}$ is then defined as the interval between its release time and deadline $\left[r_{i}^{h}, d_{i}^{h}\right)$. We define the quasi-deadline $q_{i}^{h}$ of a job $J_{i}^{h}$ is as $q_{i}^{h}=d_{i}^{h}-k C_{i}$, where $k$ is a knob that controls the ratio of execution time to deadline. We assume that the quasi-deadline control knob $k$ is a systemwide variable that the system designer determines statically and/or the system sets dynamically. We also assume that a single job of a task cannot execute in parallel.

Multiprocessor scheduling. We consider global joblevel fixed-priority preemptive scheduling on $m$ identical unit-capacity processors. In particular, we consider the EQDF scheduling algorithm that assigns priority according to quasi-deadlines; a job with an earlier quasi-deadline has 
a higher priority. Like most existing studies in multiprocessor scheduling (for example, see [12]), we assume that the system does not incur any penalty when a job is preempted or when a job is migrated from one processor to another.

Interference. The total interference on a task $\tau_{j}$ in an interval $[a, b)$ is defined by the cumulative length of all intervals in which $\tau_{j}$ is ready to execute but is not executing due to higher priority jobs of other tasks. We denote such interference with $I_{j}(a, b)$. We also define the interference $I_{j \leftarrow i}(a, b)$ of a task $\tau_{i}$ on a task $\tau_{j}$ over an interval $[a, b)$ is defined as the cumulative length of all intervals in which $\tau_{j}$ is ready to execute but it is not executing since $\tau_{i}$ is executing instead. The relation between $I_{j}(a, b)$ and $I_{j \leftarrow i}(a, b)$ is as follows:

$$
I_{j}(a, b)=\frac{\sum_{i \neq j} I_{j \leftarrow i}(a, b)}{m} .
$$

\section{EQDF Schedulability Analysis}

In this section, we derive a schedulability condition for the EQDF scheduling algorithm. We first introduce existing interference-based schedulability analysis of any work-conserving scheduling algorithms (Section 3.1), then present the worst-case interference scenarios between two tasks under EQDF scheduling (Section 3.2), compute an upper bound on the amount of execution that a task can interfere with another task on the basis of those worst-case scenarios under EQDF (Section 3.3), and finally provide a schedulability condition for EQDF scheduling (Section 3.4) and its iterative version (Section 3.5).

\subsection{Interference-based Schedulability Condition}

Schedulability analysis of global multiprocessor scheduling algorithms is presented based on the concept of worst-case interference $[7,26]$. A job of a task $\tau_{j}$ can meet its deadline, if and only if the total interference on task $\tau_{j}$ over the job's scheduling window is less than or equal to its slack time $D_{j}-C_{j}[26]$. Let $J_{j}^{*}$ denote the job instance that receives the maximal total interference among the jobs of $\tau_{j}$, and let $\bar{I}_{j}$ denote the worst-case interference on task $\tau_{j}$. Then, notice that by definition

$$
\bar{I}_{j}=\max _{h}\left(I_{j}\left(r_{j}^{h}, d_{j}^{h}\right)\right)=I_{j}\left(r_{j}^{*}, d_{j}^{*}\right) .
$$

For notational convenience, we also define

$$
\bar{I}_{j \leftarrow i}=I_{j \leftarrow i}\left(r_{j}^{*}, d_{j}^{*}\right) .
$$

With the above reasoning and notations, the necessary and sufficient schedulability condition of global multiprocessor scheduling algorithms is derived as follows $[14,26]$.

Lemma 1 (from $[14,26]$ ) A task set $\tau$ is schedulable on a multiprocessor composed by $m$ identical processors iff for each task $\tau_{j}$

$$
\sum_{i \neq j} \min \left(\bar{I}_{j \leftarrow i}, D_{j}-C_{j}+1\right)<m\left(D_{j}-C_{j}+1\right)
$$

Note that it is known to be difficult to compute $\bar{I}_{j \leftarrow i}$ precisely, so exiting approaches $[14,26]$ have used an upper bound on the interference, and therefore the test derived is changed to only a sufficient condition. Then, it needs to identify the worst-case interference scenario in which a task $\tau_{i}$ has the largest workload to interfere with a job of task $\tau_{j}$.

\subsection{Worst-Case Interference Scenarios}

In this section, we identify the worst-case interference scenarios in which the interference of a task $\tau_{i}$ on the job $J_{j}^{*}$ over the scheduling window of $J_{j}^{*}$ is maximized under EQDF scheduling.

To simplify the presentation, a job is said to be a carry-in job of an interval $[a, b)$ if it has a release time before $a$ but a deadline after $a$, a body job if it has a release time and a deadline within $[a, b)$, and a carry-out job if it has a release time within $[a, b)$ but a deadline after $b$.

Under EDF scheduling, the underlying principle behind its worst-case interference scenario is that only the carry-in and body jobs of $\tau_{i}$ over $\left[r_{j}^{*}, d_{j}^{*}\right)$ can interfere with $J_{j}^{*}$ and the interference of those jobs can be maximized when they are periodically released and they execute as late as possible (i.e., moving their deadlines as late as possible), as long as their deadlines are in the scheduling window. This is because moving their deadlines later does not affect the interference of body jobs, but it can only increase (and cannot decrease) the interference of a carry-in job [7]. Therefore, the worst-case interference scenario of $\tau_{i}$ is the one where one of its jobs has a deadline at the end of the scheduling window of $J_{j}^{*}$.

However, the above principle is not directly applicable to EQDF scheduling. Unlike EDF scheduling, even the carryout job of $\tau_{i}$ can interfere with $J_{j}^{*}$ under EQDF scheduling if the quasi-deadline of the carry-out job is earlier than or equal to that of $J_{j}^{*}$. Furthermore, the worst-case interference scenarios vary depending on the relationship between $J_{j}^{*}$ and the carry-out job.

Let us consider the situation in which jobs of $\tau_{i}$ are periodically released and one of the jobs has the same quasideadline as that of $J_{j}^{*}$. We refer to this situation as quasideadline alignment. For notational convenience, we denote by $J_{i}^{+}$the job of $\tau_{i}$ which has the same quasi-deadline as that of $J_{j}^{*}$ at quasi-deadline alignment. We refer to the difference between the deadline of $J_{j}^{*}$ and the deadline of $J_{i}^{+}$ as $\phi=d_{i}^{+}-d_{j}^{*}$. If the deadline of $J_{i}^{+}$is before that of $J_{j}^{*}(\phi \leq 0)$, the carry-out job of the scheduling window of $J_{j}^{*}$ has a lower priority than $J_{j}^{*}$, so the carry-out job cannot interfere with $J_{j}^{*}$. Therefore, for the same reason as EDF scheduling, quasi-deadline alignment is the worstcase interference scenario when $\phi \leq 0$ (depicted in Figure 1(a)). If $\phi>0$, the carry-out job can also interfere with $J_{j}^{*}$, and the interference of the job on $J_{j}^{*}$ is maximized until $\phi \leq\left(D_{i}-C_{i}\right)$. If $0<\phi \leq\left(D_{i}-C_{i}\right)$, the carry-out job corresponds with $J_{i}^{+}$and makes the maximal interference of $C_{i}$ on $J_{j}^{*}$ when it starts execution as soon as it is 


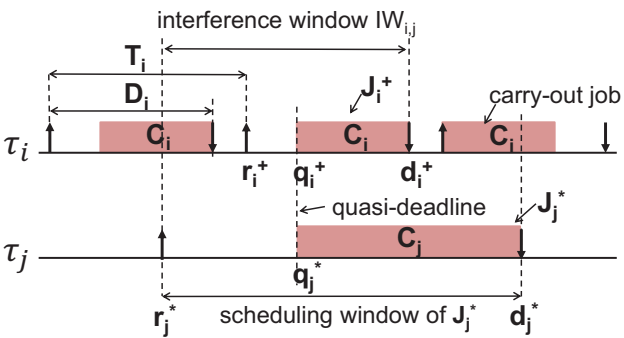

(a) Worst-case scenario I when $\phi \leq 0$.

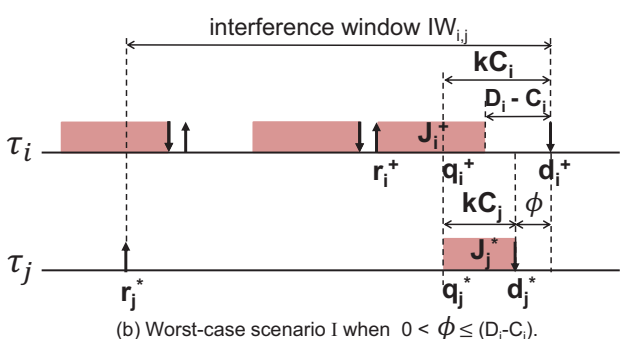

(b) Worst-case scenario I when $0<\phi \leq\left(D_{i}-C_{i}\right)$.

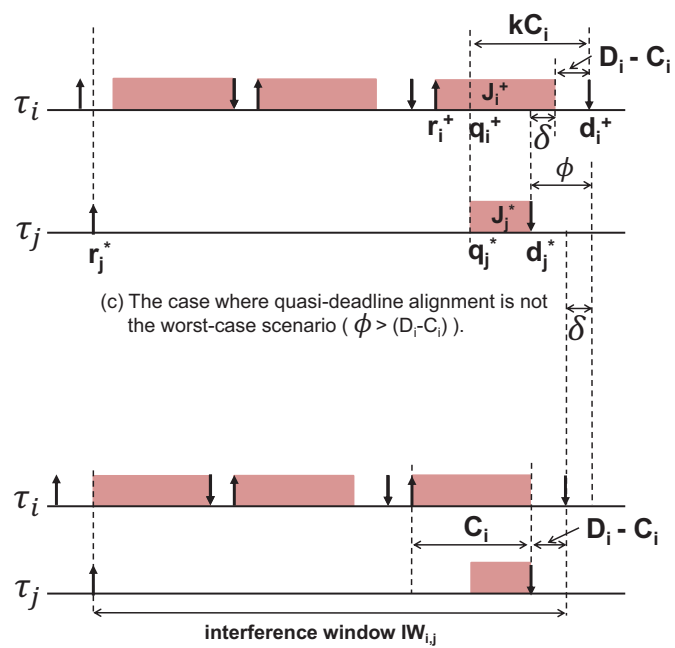

(d) Worst-case scenario II.

Figure 1. Worst-case scenarios with $k=1$

released as described in Figure 1(b). Therefore, the quasideadline assignment is the worst-case interference scenario in the case of $0<\phi \leq\left(D_{i}-C_{i}\right)$. This is because moving deadlines of $\tau_{i}$ 's jobs later until one of them meets the quasideadline of $J_{j}^{*}$ does not incur any loss of the interference of the carry-out job and does maximize the interference of both carry-in and body jobs.

However, if $\phi>\left(D_{i}-C_{i}\right)$, there can exist a situation (shown in Figure 1(c)) where the interference of the carryout job is not maximized even though the job has a higher priority than $J_{j}^{*}$ at quasi-deadline alignment. This is because the carry-out job is able to have a $\delta$ amount of execution remaining, where $\delta>0$, after the deadline of $J_{j}^{*}$ (shown in Figure 1-(c)). We can see that moving the deadlines of $\tau_{i}$ 's jobs earlier can increase (and cannot decrease) the total interference of $\tau_{i}$ on $J_{j}^{*}$, compared to that in the quasi-deadline alignment situation. This is because, firstly, moving those deadlines earlier by $\delta$ will not affect its priority order with $J_{j}^{*}$, and secondly, it will add the interference of the carry-out job into the total interference exactly as much as $\delta$ but decrease the interference of a carry-in job by at most as much as $\delta$. Thereby, in this case, the worstcase interference scenario is that the jobs of $\tau_{i}$ are periodically released in a way that one of its jobs is released exactly $C_{i}$ before the deadline of $J_{j}^{*}$ (depicted in Figure 1(d)).

To summarize, we identify two types of the worst-case interference scenarios according to the relationship between $\phi$ and $\left(D_{i}-C_{i}\right)$ at the quasi-deadline alignment situation. $J_{i}^{+}$has the same quasi-deadline as that of $J_{j}^{*}$, that is, $d_{i}^{+}-$ $k C_{i}=d_{j}^{*}-k C_{j}$, so the deadline of $J_{i}^{+}$is determined by $d_{i}^{+}=d_{j}^{*}-k C_{j}+k C_{i}$. The difference $\phi$ can be expressed in only relative terms as follow:

$$
\begin{aligned}
\phi & =d_{i}^{+}-d_{j}^{*}=d_{j}^{*}-k C_{j}+k C_{i}-d_{j}^{*} \\
& =k C_{i}-k C_{j} .
\end{aligned}
$$

We finally define two types of the worst case scenarios according to the relationship between $\phi$ and $\left(D_{i}-C_{i}\right)$.

Worst-case scenario I. In the case where it holds $\left(k C_{i}-\right.$ $\left.k C_{j}\right) \leq\left(D_{i}-C_{i}\right)$, the worst-case interference scenario is that the jobs of $\tau_{i}$ are periodically released in a way that one of the jobs has the same quasi-deadline as that of $J_{j}^{*}$ (i.e., identical with quasi-deadline alignment).

Worst-case scenario II. In the other case of $\left(k C_{i}-\right.$ $\left.k C_{j}\right)>\left(D_{i}-C_{i}\right)$, the worst-case interference scenario is that the jobs of $\tau_{i}$ are periodically released in a way that one of its jobs is released $C_{i}$ before the deadline of $J_{j}^{*}$.

In both two scenarios, the carry-in and carry-out jobs start executing as close as possible to the scheduling window of $J_{j}^{*}$ to maximize their interference.

\subsection{Bounding Interference}

For presentational convenience, we call a job of $\tau_{i}$ the last interfering job (denoted by $J_{i}^{\diamond}$ ) of $\tau_{i}$ on $J_{j}^{*}$ if the job is released latest among those that can interfere with $J_{j}^{*}$ over its scheduling window. We also define the interference window $I W_{i, j}$ of $\tau_{i}$ on $J_{j}^{*}$ as the interval from the release time of $J_{j}^{*}$ to the deadline of $J_{i}^{\diamond}$, that is, $I W_{i, j}=\left[r_{j}^{*}, d_{i}^{\diamond}\right)$. Figure 1 shows the interference window in each scenario. Note that under EDF scheduling, since the worst-case interference scenario is the one where $d_{i}^{\diamond}=d_{j}^{*}, I W_{i, j}$ is exactly the same as $\left[r_{j}^{*}, d_{j}^{*}\right)$. Under EQDF scheduling, however, $d_{i}^{\diamond}$ can be before or after, or equal to $d_{j}^{*}$.

The interference $\bar{I}_{j \leftarrow i}$ is then bounded by the largest workload of task $\tau_{i}$ in its interference window on $J_{j}^{*}$ according to the worst-case scenarios. This is because even 
though $J_{i}^{\diamond}$ can be the carry-out job of the scheduling window of $J_{j}^{*}$ (i.e., the length of the interference window is greater than that of the scheduling window of $J_{j}^{*}$ ), $J_{i}^{\diamond}$ can make the maximal interference of $C_{i}$ on $J_{j}^{*}$ under the both worst-case interference scenarios.

In the worst-case scenario $\mathrm{I}, J_{i}^{\diamond}$ has the same quasideadline as that of $J_{j}^{*}$, so the deadline $d_{i}^{\diamond}$ is determined by $d_{j}^{*}-k C_{j}+k C_{i}$. If the deadline of $J_{i}^{\diamond}$ is before the release time of $J_{j}^{*}$ (i.e., $d_{i}^{\diamond} \leq r_{j}^{*}$ ), no single job of $\tau_{i}$ can interfere with $J_{j}^{*}$ and the interference of $\tau_{i}$ on $J_{j}^{*}$ is thereby zero. Otherwise, all the jobs of $\tau_{i}$ within the interference window $\left[r_{j}^{*}, d_{i}^{\diamond}\right)$ can actually interfere with $J_{j}^{*}$. In this case, the length of the interference window $I W_{i, j}$ is computed as

$$
\begin{aligned}
\left|I W_{i, j}\right| & =d_{i}^{\diamond}-r_{j}^{*}=d_{j}^{*}-k C_{j}+k C_{i}-r_{j}^{*} \\
& =D_{j}-k C_{j}+k C_{i} .
\end{aligned}
$$

We denote by $\Phi_{i}(L)$ the maximal number of $\tau_{i}$ 's jobs that contribute with the entire execution times $\left(C_{i}\right)$ to the workload within the interval of length $L$, and it is described as

$$
\Phi_{i}(L)=\left\lfloor\frac{L}{T_{i}}\right\rfloor .
$$

The contribution of the carry-in job can then be bounded by

$$
\min \left(C_{i}, L-\Phi_{i}(L) \cdot T_{i}\right)
$$

Therefore, under the worst-case interference scenario I, the interference $\bar{I}_{j \leftarrow i}$ is bounded by

$$
\begin{aligned}
\bar{I}_{j \leftarrow i} \leq & {\left[\Phi_{i}\left(D_{j}-k C_{j}+k C_{i}\right) \cdot C_{i}+\right.} \\
& \left.\min \left(C_{i}, D_{j}-k C_{j}+k C_{i}-\Phi_{i}\left(D_{j}-k C_{j}+k C_{i}\right) \cdot T_{i}\right)\right]_{0}
\end{aligned}
$$

where it is bounded by zero when $D_{j}-k C_{j}+k C_{i}<0$.

In the worst-case scenario II, $J_{i}^{\diamond}$ is released such that its deadline $d_{i}^{\diamond}$ is equal to $d_{j}^{*}-C_{i}+D_{i}$. Then, the length of the interference window of $\tau_{i}$ on $J_{j}^{*}$ is equal to $D_{j}-C_{i}+D_{i}$. Therefore, the interference $\bar{I}_{j \leftarrow i}$ is bounded by

$$
\begin{aligned}
\bar{I}_{j \leftarrow i} \leq & \Phi_{i}\left(D_{j}-C_{i}+D_{i}\right) \cdot C_{i}+ \\
& \min \left(C_{i}, D_{j}-C_{i}+D_{i}-\Phi_{i}\left(D_{j}-C_{i}+D_{i}\right) \cdot T_{i}\right) .
\end{aligned}
$$

\subsection{EQDF Schedulability Analysis}

In the previous sub-sections, we identify the worst-case interference scenarios and compute the upper bound on the interference $\bar{I}_{j \leftarrow i}$ based on them.

We denote by $I_{j \leftarrow i}^{E Q D F}(L, k)$ an upper bound on the interference $\bar{I}_{j \leftarrow i}$ in any interval of length $L$ under the EQDF scheduling policy with a system-wide variable $k$ and described as

$$
\begin{aligned}
& I_{j \leftarrow i}^{E Q D F}(L, k)= \\
& \left\{\begin{array}{l}
{\left[\Phi_{i}\left(L-k C_{j}+k C_{i}\right) \cdot C_{i}+\right.} \\
\left.\quad \min \left(C_{i}, L-k C_{j}+k C_{i}-\Phi_{i}\left(L-k C_{j}+k C_{i}\right) \cdot T_{i}\right)\right]_{0} \\
\quad \text { if }\left(k C_{i}-k C_{j}\right) \leq\left(D_{i}-C_{i}\right) \\
\Phi_{i}\left(L+D_{i}-C_{i}\right) \cdot C_{i}+\quad \text { otherwise. }
\end{array}\right.
\end{aligned}
$$

A schedulability test for EQDF immediately follows.

Theorem 1 A task set $\tau$ is schedulable under EQDF scheduling with a system-wide variable $k$ on a multiprocessor composed by $m$ identical processors if for each task $\tau_{j}$

$$
\sum_{i \neq j} \min \left(I_{j \leftarrow i}^{E Q D F}\left(D_{j}, k\right), D_{j}-C_{j}+1\right)<m\left(D_{j}-C_{j}+1\right) .
$$

Note that the above EQDF schedulability test is a generalization of the existing EDF schedulability test [14].

\subsection{Iterative Test}

In general, bounding interference involves much pessimism, particularly, in computing the workload of a carryin job. Hence, the slack-based iterative approaches $[13,26]$ are introduced to reduce such pessimism effectively. Let $S_{j}$ denote the slack of a task $\tau_{j}$ and is defined as

$$
S_{j}=D_{j}-C_{j}-\left\lfloor\frac{\sum_{i \neq j} \min \left(\bar{I}_{j \leftarrow i}, D_{j}-C_{j}+1\right)}{m}\right\rfloor
$$

when (13) is positive. A lower bound $S_{j}^{l b}$ on the slack $S_{j}$ of a task $\tau_{j}$ is then given by

$$
S_{j}^{l b}=D_{j}-C_{j}-\left\lfloor\frac{\sum_{i \neq j} \min \left(I_{j \leftarrow i}^{E Q D F}\left(D_{j}, k\right), D_{j}-C_{j}+1\right)}{m}\right\rfloor
$$

when this term is positive.

Fortunately, the iterative test exploiting a slack value can be directly adopted into EQDF schedulability analysis. In this paper, the iterative test can be easily incorporated by replacing the bound of the carry-in job's contribution (Eq. (8)) with $\min \left(C_{i}, L-S_{i}^{l b}-\Phi_{i}(L) \cdot T_{i}\right)$.

\section{Quasi-Deadline Assignment}

In this section, we consider the problem of priority assignment under job-level fixed-priority scheduling. Specifically, we examine the $k$-controlled quasi-deadline assignment problem; given a task set, this problem finds a value of the quasi-deadline control knob $k$ such that each individual job $J_{i}^{h}$ is assigned a quasi-deadline $q_{i}^{h}$ equal to $d_{i}^{h}-k C_{i}$ and the task set is deemed schedulable under global EQDF scheduling by the schedulability test in Eq. (12). For presentational convenience, a value of $k$ is referred to as schedulable for a given task set $\tau$ if $\tau$ is deemed schedulable with this $k$ value according to Eq. (12). A solution algorithm to the $k$-controlled quasi-deadline assignment is referred to as optimal, if the solution algorithm can find any schedulable value of $k$ for a task set $\tau$ if and only if there exists some schedulable value of $k$ for $\tau$.

Section 4.1 presents an optimal solution to the problem, and Section 4.2 discusses heuristic solutions. 


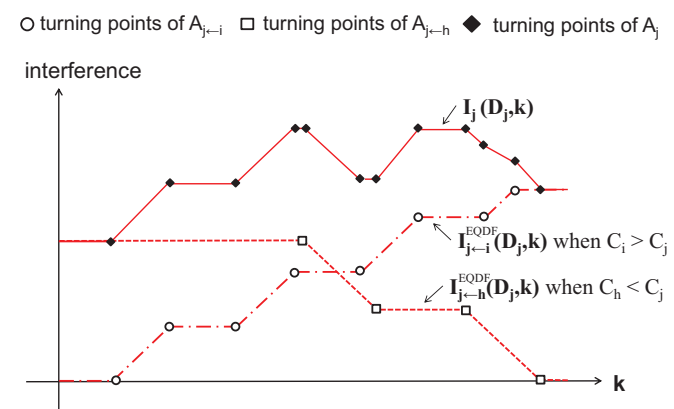

\section{Figure 2. example graph representing inter- ference made by higher priority tasks accord- ing to value $k$}

\subsection{Optimal quasi-deadline assignment}

In this section, we present an algorithm, called OQDA- $k$ (optimal quasi-deadline assignment by $k$ ), that finds a set of all the schedulable values of $k$ for a given task set. A brute-force approach would examine all possible values of $k$ for schedulability. This approach is prohibitively expensive, and it is not even applicable to the case of continuous values of $k$. Instead, we seek to identify a finite set of $k$ values that guarantees the discovery of all the schedulable values of $k$.

The OQDA- $k$ algorithm first carries out efficient discovery of a finite number of certain $k$ values (denoted by $A_{j \leftarrow i}$ ), taking advantage of interference patterns between every two tasks $\tau_{i}$ and $\tau_{j}$ (step A1). It then aggregates those $k$ values into a single set (denoted by $A_{j}$ ) per each task $\tau_{j}$ (step A2) and constructs a set of intervals (denoted by $S_{j}$ ) from $A_{j}$ for each task $\tau_{j}$ such that each interval represents a set of continuous schedulable values of $k$ for an individual $\tau_{j}$ (step A3). Finally, the algorithm generates a set of intervals (denoted by $S$ ) such that each interval contains the continuous schedulable values of $k$ for an entire task set (step A4). Algorithm 1 summarizes the OQDA- $k$ algorithm, and we describe each step in more details as follows.

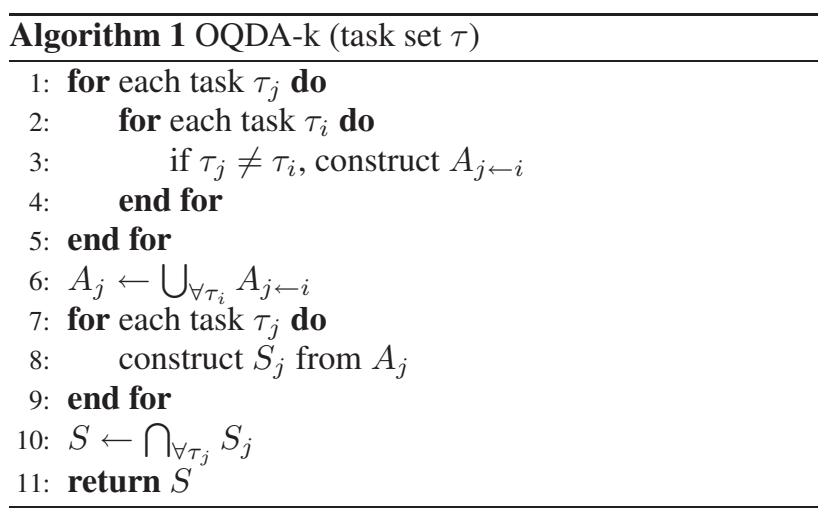

A1. In the first step A1, the algorithm generates a num- ber of discrete $k$ values for all possible pairs of tasks, exploiting the relationship between $k$ and the interference between two tasks. The interference of a task $\tau_{i}$ with a job of task $\tau_{j}$ varies with $k$ (c.f. $I_{j \leftarrow i}^{E Q D F}\left(D_{j}, k\right)$ in Eq. (11)), and $k$ makes a different impact on the interference depending on the relationship between $\tau_{i}$ and $\tau_{j}$.

It is easy to see that each job is assigned an earlier quasideadline as $k$ increases. In particular, when a task $\tau_{i}$ is has a greater execution time requirement than another $\tau_{j}$ has (i.e., $C_{i}>C_{j}$ ), an increase on $k$ results in a larger reduction to the quasi-deadline of $\tau_{i}$ than that of $\tau_{j}$. In this case, $\tau_{i}$ is then likely to have a higher priority and thus impose a greater amount of interference on $\tau_{j}$. This leads to $I_{j \leftarrow i}^{E Q D F}\left(D_{j}, k\right)$ monotonically increasing with a growing value of $k$ when $C_{i}>C_{j}$. Likewise, $I_{j \leftarrow i}^{E Q D F}\left(D_{j}, k\right)$ decreases monotonically as $k$ increases if $C_{i}<C_{j}$. When $C_{i}=C_{j}$, on the other hand, $I_{j \leftarrow i}^{E Q D F}\left(D_{j}, k\right)$ remains constant because the quasi-deadlines of $\tau_{i}$ and $\tau_{j}$ and their priorities remain relatively the same even though $k$ varies.

Figure 2 illustrates the impact of $k$ on $I_{j \leftarrow i}^{E Q D F}\left(D_{j}, k\right)$. A value of $k$ is said to be a turning point of $I_{j \leftarrow i}^{E Q D F}\left(D_{j}, k\right)$ if $I_{j \leftarrow i}^{E Q D F}\left(D_{j}, k\right)$ changes its slope at this $k$ value. The formula $I_{j \leftarrow i}^{E Q D F}\left(D_{j}, k\right)$ defined in Eq. (11) is a combination of linear and constant shape functions as shown in Figure 2. By definition, $I_{j \leftarrow i}^{E Q D F}\left(D_{j}, k\right)$ is lower-bounded by zero, and it is also upper-bounded by the amount of the interference in the worst-case scenario II. This is because the condition of $k C_{i}-k C_{j} \leq\left(D_{i}-C_{i}\right)$ (c.f. Eq. (11)) makes the interference in the worst-case scenario I always lower than or equal to that in the worst-case scenario II.

When $I_{j \leftarrow i}^{E Q D F}\left(D_{j}, k\right)$ is a monotonically increasing function, it starts from the lower-bound point increasing linearly as $k$ increases. It then becomes staying constant while the contribution of the carry-in job is bounded by $C_{i}$ and the number of body and carry-out jobs does not change as $k$ increases. It resumes increasing linearly again when a new body comes in. The process of increasing linearly and staying constant repeats until it reaches the upper-bound point. Therefore, $I_{j \leftarrow i}^{E Q D F}\left(D_{j}, k\right)$ has a finite number of turning points. We can then easily calculate all turning points with this understanding of the dynamics of $I_{j \leftarrow i}^{E Q D F}\left(D_{j}, k\right)$.

Let $A_{j \leftarrow i}$ denote a set of all turning points of $I_{j \leftarrow i}^{E Q D F}\left(D_{j}, k\right)$. In this step, the OQDA- $k$ algorithm constructs $A_{j \leftarrow i}$ for all tasks $\tau_{i}$ and $\tau_{j}$.

A2. The previous step looked at the relationship between $k$ and the interference of a single task $\tau_{i}$ on $\tau_{j}$, and this step explores the relationship between $k$ and a total interference imposed on $\tau_{j}$ by an entire task set. Let us define $I_{j}\left(D_{j}, k\right)$ as the sum of individual interferences $\left(I_{j \leftarrow i}^{E Q D F}\left(D_{j}, k\right)\right)$. It is also a combination of linear and constant shape functions. Then, $I_{j}\left(D_{j}, k\right)$ also has a sequence of turning points. Let $A_{j}$ denote a set of whole turning points of $I_{j}\left(D_{j}, k\right)$. If a value of $k$ is a turning point of $I_{j \leftarrow i}^{E Q D F}\left(D_{j}, k\right)$, then it is also a turning point of $I_{j}\left(D_{j}, k\right)$. So we can construct $A_{j}$ as the 
union of all the turning points of $I_{j \leftarrow i}^{E Q D F}\left(D_{j}, k\right)$ for all tasks $i \neq j$. Even though $I_{j}\left(D_{j}, k\right)$ is a combination of linear and constant functions, unlike $I_{j \leftarrow i}^{E Q D F}\left(D_{j}, k\right), I_{j}\left(D_{j}, k\right)$ does not increase (or decrease) monotonically over all $k$ values. However, $I_{j}\left(D_{j}, k\right)$ remains constant or increase (or decrease) linearly within an interval between two consecutive turning points.

A3: How to find schedulable $k$. Recall that a value of $k$ is schedulable for a task $\tau_{j}$ if $\tau_{j}$ is deemed schedulable with this $k$ value according to Eq. (12). The third step finds out all the schedulable values of $k$ (denoted by $S_{j}$ ) out of a set of turning points $\left(A_{j}\right)$ for each task $\tau_{j}$. Consider two consecutive turning points of $\tau_{j}\left(p_{j}^{h} \in A_{j}\right.$ and $\left.p_{j}^{h+1} \in A_{j}\right)$. That is, there does not exist $p^{\prime} \in A_{j}$ such that $p_{j}^{h}<p^{\prime}<$ $p_{j}^{h+1}$. We consider four cases in constructing $S_{j}$ with $p_{j}^{h}$ and $p_{j}^{h+1}$ according to their schedulability.

Firstly, if both $p_{j}^{h}$ and $p_{j}^{h+1}$ are schedulable for $\tau_{j}$, then all the values of $k$ within the interval $\left[p_{j}^{h}, p_{j}^{h+1}\right]$ are also schedulable. This is because $I_{j}\left(D_{j}, k\right)$ remains constant or moves linearly within the two consecutive turning points. So, the interval $\left[p_{j}^{h}, p_{j}^{h+1}\right]$ is added into $S_{j}$. Secondly, if neither $p_{j}^{h}$ nor $p_{j}^{h+1}$ is schedulable, there is no schedulable value of $k$ within the interval $\left[p_{j}^{h}, p_{j}^{h+1}\right]$ and nothing is added into $S_{j}$. Thirdly, if $p_{j}^{h}$ is schedulable but $p_{j}^{h+1}$ is not, there must exist $k^{\prime} \in\left[p_{j}^{h}, p_{j}^{h+1}\right]$ such that $\left[p_{j}^{h}, k^{\prime}\right]$ is the interval of schedulable values of $k$ but $\left(k^{\prime}, p_{j}^{h+1}\right]$ is not. Then, $\left[p_{j}^{h}, k^{\prime}\right]$ is inserted into $S_{j}$. In the fourth case where $p_{j}^{h}$ is not schedulable but $p_{j}^{h+1}$ is, another interval is added into $S_{j}$ in a similar way to the third case.

A4. The four step finally constructs a set of all the schedulable values of $k$ (denoted by $S$ ) for a given task set $\tau$. The set $S$ is indeed the intersection of all $S_{j}$. For each element $s \in S$, there exists $s_{j} \in S_{j}$ for all tasks $\tau_{j}$ such that $s \in s_{j}$.

\section{Theorem 2 The OQDA- $k$ algorithm is optimal.}

Proof. We show this theorem by contradiction. Suppose the set $S$ computed by the OQDA- $k$ algorithm is empty even though there exists a schedulable value of $k$ (denoted as $k^{*}$ ). We consider two cases depending on whether $k^{*}$ is a turning point.

Suppose $k^{*}$ is a turning point of the interference function $I_{j \leftarrow i}^{E Q D F}\left(D_{j}, k\right)$ for tasks $\tau_{i}$ and $\tau_{j}$. According to the OQDA- $k$ algorithm, $k^{*}$ is then placed into $A_{j \leftarrow i}$ (i.e., line 3 in Algorithm 1) and subsequently included in $A_{j}, S_{j}$, and $S$ (i.e., lines 6, 8, and 10 in Algorithm 1). This contradicts the assumption that $S$ is empty.

Consider the other case where $k^{*}$ is not a turning point. Then, there exist two consecutive turning points of $A_{j}$ (denoted as $p_{j}^{h}$ and $p_{j}^{h+1}$ ) such that $p_{j}^{h}<k^{*}<p_{j}^{h+1}$. From the assumption that $S$ is empty, neither $p_{i}^{h}$ nor $p_{j}^{h+1}$ is schedulable. Otherwise, any schedulable turning point should be added into $S_{j}$ and thereby into $S$, contradicting the assumption of the empty $S$. So, $p_{i}^{h}$ and $p_{j}^{h+1}$ must be not schedulable. Then, $k^{*}$ should not be schedulable either, since $I_{j \leftarrow i}^{E Q D F}\left(D_{j}, k\right)$ is constant or linear within the interval $\left[p_{i}^{h}, p_{j}^{h+1}\right]$. This contradicts the assumption that $k$ is schedulable. This concludes the proof of Theorem 2 .

Complexity. We denote the number of tasks in a task set by $n$. For each task $\tau_{j}$, the OQDA- $k$ algorithm performs as many schedulability tests as $\left|A_{j}\right|$ at most. The complexity of constructing $S$ as the intersection of all $S_{j}$ is $O\left(n \cdot\left|S_{j}\right|^{2}\right)$. Since $\left|S_{j}\right| \leq\left|A_{j}\right|$, the running time of this algorithm is thereby $O\left(n \cdot\left|A_{j}\right|^{2}\right)$.

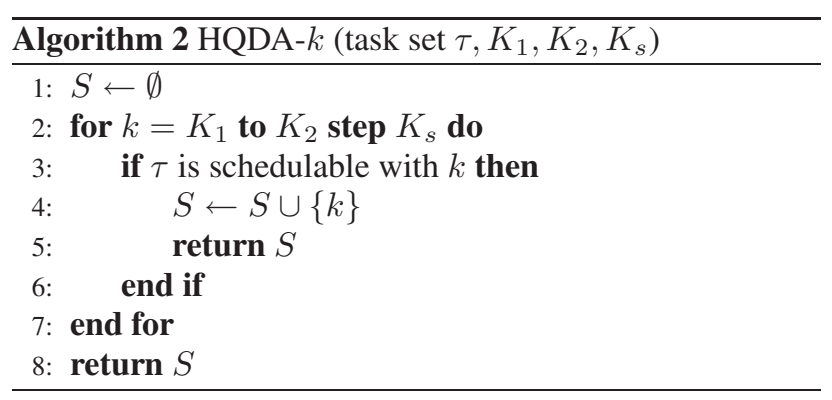

\subsection{Heuristic Priority Assignment}

The OQDA- $k$ algorithm identifies and explores all the turning points of an entire task set to find out all optimal values of $k$. As the number of tasks increases, the optimal algorithm has a fast growing number of turning points to explore and thereby its running time also increases rapidly. Thus, the OQDA- $k$ algorithm is good for optimal quasideadline assignment at design time. However, it may not be appropriate for reconfiguring quasi-deadlines dynamically at run-time.

Therefore, we introduce a heuristic algorithm (HQDA$k$ ) that finds a schedulable value of $k$ in a sub-optimal but efficient way. Algorithm 2 summarizes this heuristic algorithm. This algorithm is given a set of $k$ values and repeats the process of examining whether there exists a schedulable value of $k$ in the given set until it finds any solution or there is no more element in the set to examine. The set is given as an interval $\left[K_{1}, K_{2}\right]$ with a stepsize $K_{s}$, and the algorithm checks with $k=K_{1}, K_{1}+K_{s}, K_{1}+2 K_{s}, \cdots, K_{2}$. The evaluation of this heuristic algorithm is provided in the next section.

\section{Experimental results}

This section presents simulation results to evaluate the proposed EQDF schedulability tests and quasi-deadline assignment algorithms.

Simulation environment. Task sets are generated based on a technique proposed earlier [6], which has also been used in many previous studies (e.g., see [2, 26, 21]). We 


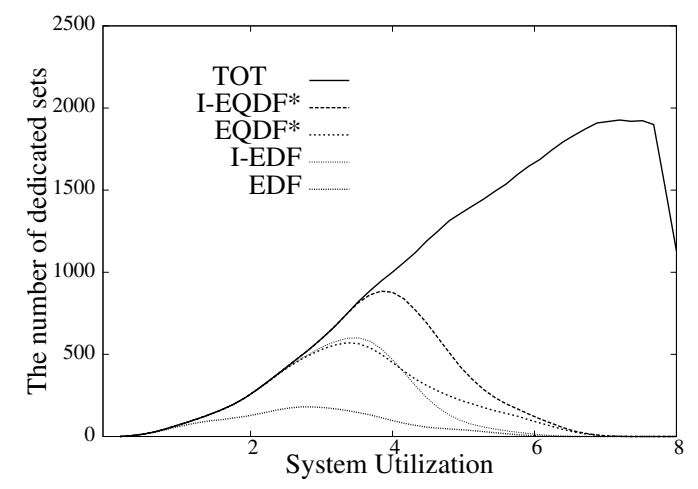

Figure 3. Schedulability of EDF and EQDF

have two input parameters. One is the number of processors ( $m=4$ or 8 ), and the other is a task utilization parameter. For each task $\tau_{i}, T_{i}$ is uniformly chosen in $[100,1000]$, and $C_{i}$ is chosen based on a bimodal or exponential task utilization parameter ${ }^{2}$. Although our proposed EQDF analysis is applicable to both implicit and constraint deadline models, because of page limit, we only show the results of the implicit deadline model: $D_{i}$ is set equal to $T_{i}$. For each task utilization model, we repeat the following procedure to generate 1,000 task sets.

1. Initially, we generate a set of $m+1$ tasks.

2. We check whether the generated task set can pass a necessary feasibility condition $[8,11]$.

3. If it fails to pass the feasibility test, we discard the generated task set and return to Step 1. Otherwise, we include this set for evaluation. Then, this set is used as a basis for the next task set; we create a new set by adding a new task into the old set and return to Step 2 .

For any given $m$, we create 1,000 task sets for an individual task utilization model, resulting in 10,000 task sets in total.

EDF vs. EQDF. Our first simulations were run to compare the EQDF schedulability tests derived in this paper with the existing tests of the only, well-known job-level fixed-priority (JFP) scheduling algorithm, EDF. Aiming at seeing how effectively the schedulability of JFP scheduling can improve with EQDF, the quasi-deadline control knob $k$ is set to an optimal value for each task set through the OQDA- $k$ algorithm. The following schedulability tests were considered:

- the EDF test in [26] (EDF)

- the iterative EDF test in [26] (I-EDF)

- our EQDF test in Theorem 1 with an optimal value $k^{*}$ $\left(\mathrm{EQDF}^{*}\right)$

- our iterative EQDF test in Eq. (14) with an optimal value $k^{*}\left(\mathrm{I}-\mathrm{EQDF}^{*}\right)$

\footnotetext{
${ }^{2}$ For a given bimodal parameter $p$, a value for $C_{i} / T_{i}$ is uniformly chosen in $[0,0.5)$ with probability $p$, where $p=0.1,0.3,0.5,0.7$, or 0.9 For a given exponential parameter $1 / \lambda$, a value for $C_{i} / T_{i}$ is chosen according to the exponential distribution whose probability density function is $0.5 \cdot \lambda \cdot \exp (-\lambda \cdot x)$, where $\lambda=0.1,0.3,0.5,0.7$ or 0.9 .
}

Figure 3 compares EDF and EQDF on the basis of schedulability with $m=8$. Each line represents the number of task sets deemed schedulable by one specific test, except that the curve labeled with TOT; it represents an upper bound on the feasible task sets. Figure 3 shows that EQDF* significantly outperforms EDF. In particular, I-EQDF* dominates all the other tests, making significant differences when the system utilization is between $m / 3$ and $2 m / 3$. Table 1 also shows that EQDF* finds $160 \%-320 \%$ more task sets schedulable than EDF does, and I-EQDF* detects $40 \%$-60\% more task sets schedulable than I-EDF does on 4 and 8 processors, respectively. Our simulation results indicate that the schedulability of JFP scheduling can improve significantly when the priorities of individual jobs are well assigned. That is, it is important to determine a good value of $k$ for the effectiveness of EQDF scheduling. The OQDA- $k$ algorithm is able to find an optimal value of $k$. However, as shown in Table 1, it is computationally expensive. Its running time is four orders of magnitude greater than that of EDF tests, leaving the OQDA- $k$ algorithm inappropriate for online priority assignment. This entails good, cost-effective, alternative solutions to online quasi-deadline assignment.

\begin{tabular}{c|c|c|c|c|c}
\hline & $m$ & EDF & I-EDF & EQDF $^{*}$ & I-EQDF \\
\hline Schedulability & 4 & 11.1 & 26.7 & 30.4 & 37.8 \\
$(\%)$ & 8 & 6.5 & 18.3 & 20.7 & 29.1 \\
\hline Running & 4 & 0.4 & 5.3 & $5.4 \times 10^{3}$ & $1.42 \times 10^{5}$ \\
Time $(\mu \mathrm{s})$ & 8 & 0.7 & 23.4 & $2.26 \times 10^{4}$ & $1.85 \times 10^{6}$ \\
\hline
\end{tabular}

Table 1. EDF and EQDF tests

Characterizing schedulable $k$ values. We seek to understand the impact of $k$ on schedulability in order to gain good insights towards online quasi-deadline assignment.

To simply the presentation, we define $N(a, b)$ as the number of task sets in which there exists a schedulable value $k^{*} \in[a, b]$. We also define $F(a, b)$ as the ratio of the number of task sets that have a schedulable value $k^{*} \in[a, b]$ to the number of schedulable task sets with any schedulable value $k^{*} \in[-\infty, \infty]$. That is, $F(a, b)$ is defined as the ratio of $N(a, b)$ to $N(-\infty, \infty)$.

Figure 4 shows how schedulable values $k^{*}$ are distributed over $k$ on $m=8$ processors. More specifically, Figure 4(a) plots $F\left(-\infty, k^{\prime}\right)$, representing the cumulative distribution of schedulable $k$ values in a direction from $-\infty$ to the value of $k^{\prime}$. On the other hand, Figure 4(b) plots $F\left(k^{\prime}, \infty\right)$ in the other direction from $\infty$ to $k^{\prime}$. Each curve represents the cumulative distribution of one specific task utilization model (with either a bimodal or exponential parameter). It is consistently shown across those 10 different task utilization models that there is a sharp increase in a short range of $k^{\prime}$ values before and after zero, reaching at the 95th percentile or higher. This implies that we can find a schedulable value $k^{*}$ with a high probability by looking at a short range of $k$ values, instead of exploring the whole range of $[-\infty, \infty]$, if any schedulable value $k^{*}$ exists. 


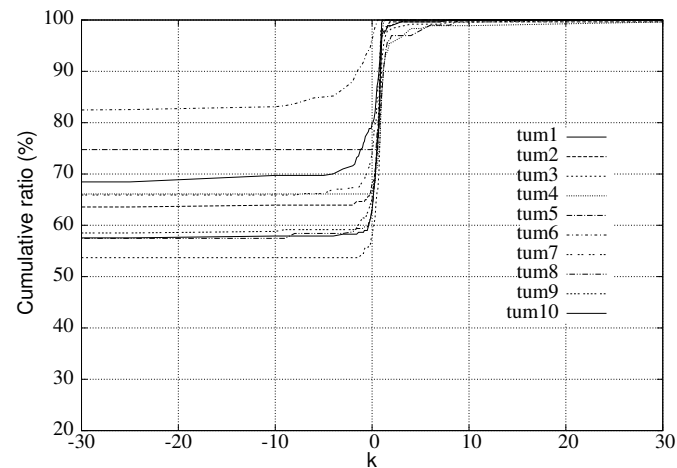

(a) $F(-\infty, k)$

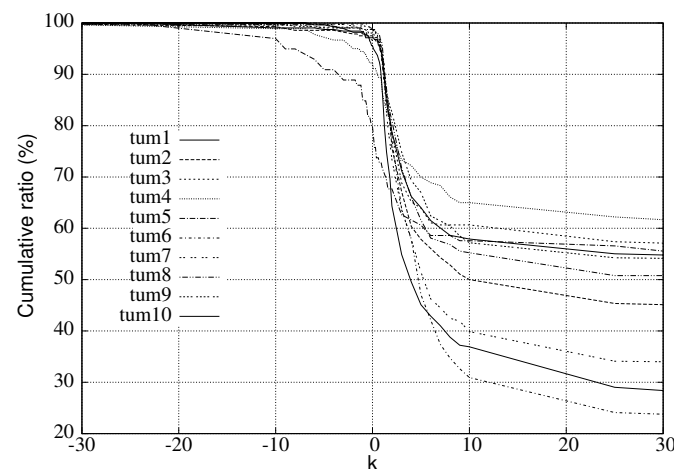

(b) $F(k, \infty)$

Figure 4. Cumulative distribution of schedulable $k$ values on $m=8$ processors

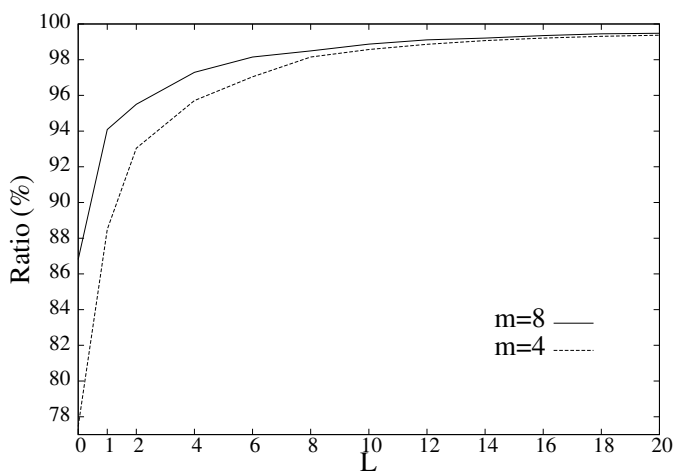

Figure 5. Ratio of schedulable task sets with $\left[t^{*}, t^{*}+L\right]$ to those with $[-\infty, \infty]$

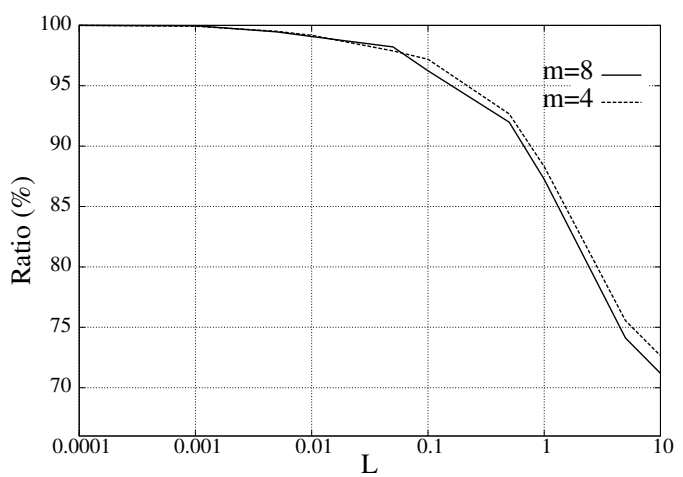

\section{Figure 6. Ratio of the size of $S(L)$ to the size of $S$}

This motivates investigation into how likely a schedulable value of $k$ belongs to an interval of length $L$. For a given length $L$, we find a real value $t^{*}$ such that $N\left(t^{*}, t^{*}+L\right)$ is maximized. Figure 5 plots $F\left(t^{*}, t^{*}+L\right)$ over different values of $L$ with $m=4$ and $m=8$, and Table 2 shows corresponding intervals $\left[t^{*}, t^{*}+L\right]$. It is shown that even a single value of $k$, such as $k=0.7$ on $m=4$ and $k=1$ on $m=8$, makes a schedulable value $k^{*}$ with a higher probability than $78 \%$ and $87 \%$, respectively. Such a probability increases sharply with a small value of $L$ and grows slowly going beyond the 99th percentile when $L$ is close to 20. This presents a good intuition into how long an interval of interest should be enough to include a schedulable value of $k$ with a certain degree of probability.

\begin{tabular}{c|c|c|c|c|c}
\hline \multirow{2}{*}{$m$} & \multicolumn{5}{|c}{ Interval length $(L)$} \\
\cline { 2 - 6 } & 0 & 1 & 4 & 8 & 16 \\
\hline 4 & {$[0.7,0.7]$} & {$[0.2,1.2]$} & {$[-2.4,1.6]$} & {$[-4.9,3.1]$} & {$[-9.0,7.0]$} \\
\hline 8 & {$[1.0,1.0]$} & {$[0.3,1.3]$} & {$[-2.5,1.5]$} & {$[-5.3,2.7]$} & {$[-10.8,5.2]$} \\
\hline
\end{tabular}

Table 2. Intervals $\left(\left[t^{*}, t^{*}+L\right]\right)$

Table 2 show where those intervals $\left[t^{*}, t^{*}+L\right]$ are located, and this provides an idea into which interval of $k$ should be examined for the efficient discovery of schedulable $k$ value. We are then interested in how densely to sample a given interval. Recall that the OQDA- $k$ algorithm generates a set $S$ that contains all schedulable values of $k$ for a given task set. Each element $s \in S$ is an interval that holds a series of continuous $k$ schedulable values. Let us define $S(L)$ as a subset of $S$ such that $S(L)$ includes $s \in S$ if $|s| \geq L$. Figure 6 shows the ratio of the size of $S(L)$ to the size of $S$. As shown in the figure, every interval $s \in S$ has a length greater than or equal to 0.001 , and more than $99 \%$ of the intervals of $S$ have a length greater than or equal to 0.01 . The percentage drops significantly when $L$ becomes larger than 0.1 . This gives an insight into how densely our heuristic algorithm samples a given interval to locate a schedulable value of $k$.

\begin{tabular}{c|c|c|c|c|c|c}
\hline & \multirow{2}{*}{} & \multicolumn{6}{|c}{ Length (L) \& Interval } \\
\cline { 3 - 7 } & & 0 & 4 & 16 & 32 & 64 \\
& & {$[1,1]$} & {$[-2,2]$} & {$[-8,8]$} & {$[-16,16]$} & {$[-32,32]$} \\
\hline Schedulability & 4 & 77.4 & 95.1 & 98.2 & 98.7 & 99.2 \\
ratio (\%) & 8 & 86.6 & 96.9 & 98.6 & 99.1 & 99.2 \\
\hline Running time & 4 & 0.3 & 2.7 & 12.2 & 24.4 & 47.4 \\
ratio $\left(10^{-3}\right)$ & 8 & 0.06 & 0.6 & 4.1 & 5.1 & 15.6 \\
\hline
\end{tabular}

Table 3. The ratio of heuristics to optimal 
Optimal vs. heuristic solutions. Based on understanding of the characteristics of optimal solutions, we determine where and how densely the HQDA- $k$ algorithm examines $k$ values. Table 3 shows the interval that HQDA- $k$ examines for a given value of $L$, and we set the sampling step to 0.1. For example, when $L=4$, HQDA- $k$ examines the interval $[-2,2]$ with the step size of 0.1 . This way, we can effectively reduce the search space of HQDA- $k$. Table 3 also shows that the ratio of heuristic solutions to optimal in terms of schedulability and running time. It is shown that when $L=4$, the heuristic algorithm finds a solution $95 \%$ close to optimal with a shorter running time by three to four orders of magnitude. This translates into that the heuristic solution has a comparable running time with EDF analysis but produces $40 \%-57 \%$ better results than EDF. When $L=64$, heuristic solutions have only less than $1 \%$ loss of optimality reducing running time by two orders of magnitude on 4 and 8 processors, respectively.

\section{Conclusion}

In this paper, we presented the EQDF algorithm (categorized into job-level fixed-priority (JFP) scheduling) to overcome poor performance of the existing JFP algorithm on multiprocessor platforms. EQDF assigns priority to jobs according to their quasi-deadlines $\left(d_{i}-k C_{i}\right)$, and the control knob $k$ allows to balance efficiently between urgency and parallelism in quasi-deadline assignment. We also presented an optimal solution to the quasi-deadline assignment subject to the proposed EQDF schedulability analysis for design time, and a heuristic solution for runtime. We performed an extensive empirical study to gain good insights into how the search space of the heuristic solution can be effectively reduced. Based on our understanding of empirical results, we can reduce the running time of our proposed heuristic algorithm significantly (two to four orders of magnitude) at the expense of $1 \sim 5 \%$ optimality loss.

In this paper, the notion of quasi-deadline has been explored for job-level fixed-priority scheduling. We plan to extend this notion towards job-level dynamic-priority algorithms such as EDZL.

\section{Acknowledgements}

This work was supported in part by the IT R\&D Program of MKE/KEIT [2011-KI002090], Basic Research Laboratory (BRL) Program (2009-0086964), Basic Science Research Program (2011-0005541), and NCRC (20110018245) through the National Research Foundation of Korea (NRF) funded by the Korea Government (MEST), and KAIST-Microsoft Research Collaboration Center.

\section{References}

[1] B. Andersson. Global static-priority preemptive multiprocessor scheduling with utilization bound 38\%. In ICPDS, 2008.

[2] B. Andersson, K. Bletsas, and S. Baruah. Scheduling arbitrarydeadline sporadic task systems on multiprocessor. In RTCSA, 2008.

[3] B. Andersson and J. Jonsson. Fixed-priority preemptive multiprocessor scheduling: To partition or not to partition. In RTCSA, 2000.

[4] N. Audsley. Optimal priority assignment and feasibility of static priority tasks with arbitrary start times. Technical Report YCS164, Department of Computer Science, University of York, 1991.

[5] N. Audsley. On prioirty assignment in fixed priority scheduling. Information Processing Letters, 79(1):39-44, 2001.

[6] T. Baker. An analysis of EDF schedulability on a multiprocessor. IEEE Transactions on Parallel Distributed Systems, 16(8):760-768, 2005.

[7] T. P. Baker. Multiprocessor EDF and deadline monotonic schedulability analysis. In RTSS, 2003.

[8] T. P. Baker and M. Cirinei. A necessary and sometimes sufficient condition for the feasibility of sets of sporadic hard-deadline tasks. In RTSS, pages 178-190, 2006.

[9] T. P. Baker, M. Cirinei, and M. Bertogna. EDZL scheduling analysis. Real-Time Systems, 40:264-289, 2008.

[10] S. Baruah. Techniques for multiprocessor global schedulability analysis. In RTSS, 2007.

[11] S. Baruah, V. Bonifaci, A. Marchetti-Spaccamela, and S. Stiller. Implementation of a speedup-optimal global EDF schedulability test. In ECRTS, 2009.

[12] S. Baruah, N. K. Cohen, C. G. Plaxton, and D. A. Varvel. Proportionate progress: a notion of fairness in resource allocation. Algorithmica, 15(6):600-625, 1996.

[13] M. Bertogna and M. Cirinei. Response-time analysis for globally scheduled symmetric multiprocessor platforms. In RTSS, 2007.

[14] M. Bertogna, M. Cirinei, and G. Lipari. Improved schedulability analysis of EDF on multiprocessor platforms. In ECRTS, 2005.

[15] M. Bertogna, M. Cirinei, and G. Lipari. New schedulability tests for real-time task sets scheduled by deadline monotonic on multiprocessors. In ICPDS, 2005.

[16] R. Davis and A. Burns. Priority assignment for global fixed priority pre-emptive scheduling in multiprocessor real-time systems. In RTSS, 2009.

[17] R. I. Davis and A. Burns. FPZL schedulability analysis. In RTAS, 2011.

[18] M. L. Dertouzos and A. K. Mok. Multiprocessor on-line scheduling of hard-real-time tasks. IEEE Transactions on Software Engineering, 15:1497-1506, 1989.

[19] J. Goossens, S. Funk, and S. Baruah. Priority-driven scheduling of periodic task systems on multiprocessors. Real-Time Systems, 25(2-3):187-205, 2003.

[20] J. Lee, A. Easwaran, and I. Shin. LLF Schedulability Analysis on Multiprocessor Platforms. In RTSS, 2010.

[21] J. Lee, A. Easwaran, and I. Shin. Maximizing contention-free executions in multiprocessor scheduling. In RTAS, 2011.

[22] S. K. Lee. On-line multiprocessor scheduling algorithms for realtime tasks. In IEEE Region 10's Ninth Annual International Conference, 1994.

[23] J. Leung and J. Whitehead. On the complexity of fixed-priority scheduling of periodic real-time tasks. Performance Evaluation, $2: 237-250,1982$

[24] C. Liu and J. Layland. Scheduling algorithms for multiprogramming in a hard-real-time environment. Journal of the ACM, 20(1):46-61, 1973.

[25] J. Liu. Real-time systems. Prentice Hall, 2000.

[26] M. Bertogna, M. Cirinei, and G. Lipari. Schedulability analysis of global scheduling algorithms on multiprocessor platforms. IEEE Transactions on Parallel and Distributed Systems, 20:553566, 2009. 